\title{
EFFECT OF HIGH LEVELS OF NITROGEN AND PHOSPOURS FERTILIZER, PINCHING, AND SEED RATE ON GROWTH AND YIELD COMPONENTES OF Nigella sativa $\mathrm{L}$. 1-VEGETATIVE GROWTH AND SEED YIELD \\ Yousif H. Hammo \\ Hort. Dept., College of Agriculture, Dohuk Univ., Iraq
}

\begin{abstract}
Field experiment was conducted during the season 2005-2006 in Singar - Mosul city to investigate the effects of high level $\left(280 \mathrm{~N}, 260 \mathrm{P}_{2} \mathrm{O}_{5}\right) \mathrm{kg} \mathrm{ha}^{-1}$ and very high level $\left(320 \mathrm{~N}, 300 \mathrm{P}_{2} \mathrm{O}_{5}\right) \mathrm{kg} \mathrm{ha}^{-1}$ fertilizer, pinch and without pinch, and plant seed rate sowing $(0.6,0.8,1.0,1.2) \mathrm{g} / 10 \mathrm{~m}^{2}$ cultivated within 3 , $4,5,6$ rows respectively in (10) $\mathrm{m}^{2}$ plot area on growth and yield of Nigella sativa L. The experiment was laid out in randomized complete block design with three replications. The results indicated that. Very high level of nitrogen and phosphorus caused a significant increase in plant length, stem diameter, fresh weight, plant seed yield and total seeds yield $\mathrm{kg} / \mathrm{ha}$, were as branches number, and fruits number cannot be effected significantly by previous factor. Pinching causes a significantly increased in branches number, fruits number, while plant high decreased significantly. Increased seed rate sowing from 0.6 to $1.2 \mathrm{~g} / 10 \mathrm{~m}^{2}$ caused a significant increasing in branches number, fresh weight, and plant seeds yield, while stem diameter, and fruits number didn't effected significantly by this factor except total seeds yield $\mathrm{kg} / \mathrm{ha}$ which increased significantly when seed rate sowing are increased to $1.2 \mathrm{~g} / 10 \mathrm{~m}^{2}$ and they reach $651.85,843.56,1076.51,1232.67 \mathrm{~kg} /$ ha for the four seed rate consecutively.
\end{abstract}

\section{INTRODUCTION}

Nigella (Nigella sativa L.) is an annual herbaceous plant belonging to the family Ranunculacea. (Majed and Mahmod, 1988). It is grown in Mediterranean region and widely cultivated throughout Syria, Egypt, Iraq, Iran, and Turkey (Al-Dagawi, 1996). Mature seeds are consumed for edible purposes as seasoning for vegetables, legumes and different types of baked products (Atta, 2003). It has been used as herbal medicine for more than 2000 years such as bronchodilator, (El-Kadi and Kandil, 1987), antibacterial (Hanafy and Hatem, 1991). Also it used In folk medicine as a natural stimulant of immunity, anti-inflammatory cough, analgesic, diuretic, anti-diabetic, (AlDagawi, 1996; Tierra, 2002). To realize the full yield potential of Nigella many agricultural practices will have to be optimized for its yield. Seed rate is the key factor determining effecting the yield and yield components. Ahmed (1997) show that there were no significant effect in plant high, branch number when increasing planting distant from $20-40 \mathrm{~cm}$ between nigella plants, while $30 \mathrm{~cm}$ caused an increment in vegetative dry weight, fruit number, and seed yield per plant. Al-khatony (2003) on Nigella sativa found that increasing plants distance from 15 to $35 \mathrm{~cm}$ had significant effects on seed components except fixed and volatile oil which significantly decrease. 
Received 15/8/2007 accepted 21/11/2007.

Hand pinching is a common floricultural practice (Ecke and Matkin, 1976) designed to encourage auxiliary shoot development and increasing flower number for display purposes (Love, 1975; Larson, 1980). Phetpradap et al. (1994) found that hand Pinching of Dahlias had no significant effect on lateral branch length, seed yield of plant but reduced the spread of flowering. Al-khatony (2003) on Nigella sativa obtained that pinching increase stem diameter, branches number, vegetative dry weight, fruit numbers, plant production, seed yield, essential, and volatile oil production with $8.1 \%$, $6.1 \%, 14.9 \%, 11.4 \%, 8.1 \%, 7.4 \%, 7.0 \%, 5.8 \%$ when compared with without pinch. The other important factor is nitrogen and Phosphorus which is a major component an effect on aspects of growth and metabolism then in plants yields (Wikipedia, 2007). Abu Zaid (1986) mentioned that nigella plants need high quantity of phosphorus and potassium fertilized and he recommended to add (200) $\mathrm{kg}$ supper phosphate /Feddan $\left(4200 \mathrm{~m}^{2}\right)$. Ahmed (1997) found that foliar Nigella sativa plants with triple calcium super phosphate fertilizer $(20,40,60) \mathrm{P}_{2} \mathrm{O}_{5}$ /feddan caused a significant increase in plant high, stem diameter, branch numbers, dry weight, fruit number. Singh et al. (1999) found that increasing nitrogen fertilizer levels $(0,30$, and 60) $\mathrm{kg}$ $\mathrm{N} \mathrm{ha}^{-1}$ increased the seed yield production of nigella plants. Hammo and AlAtrakchii (2006) on Nigella sativa found that increase fertilizer level of nitrogen and phosphorus to $240 \mathrm{~N}$ and $220 \mathrm{~kg} \mathrm{P}_{2} \mathrm{O}_{5} \mathrm{~kg} \mathrm{ha}^{-1}$ causes significant increases in plant high, stem diameter, branches number, vegetative dry weight, fruits number, and seed production. This study were done to clarify the influence of very high levels of fertilizers, seed rate sowing, and pinching on vegetative growth and seed yield of nigella plant.

\section{MATERIALS AND METHODS}

Field experiment was carry out during the season 2005-2006 in Singar/ Mosul city to investigate the effects of some agricultural factors on vegetative growth and seed yield of Nigella sativa L. Seeds which were obtained from the herbal market in Mosule was cultivated at $10^{\text {th }}$ Oct 2005 with Sowing rate of $(0.6,0.8,1.0,1.2) \mathrm{g} /$ plot $4 \times 2.5 \mathrm{~m}\left(10 \mathrm{~m}^{2}\right)$ by hand within $3,4,5,6$ rows for each seed rate respectively, pinch and without pinch was done after 3-4 pear of leaves emerge on plant. Fertilize with two level of nitrogen and phosphorus high level $\left(280 \mathrm{~N}, 260 \mathrm{P}_{2} \mathrm{O}_{5}\right) \mathrm{kg} \mathrm{ha}^{-1}$ and very high level $\left(320 \mathrm{~N}, 300 \mathrm{P}_{2} \mathrm{O}_{5}\right) \mathrm{kg} \mathrm{ha}^{-1}$ added to plant at two time, the half amount of nitrogen and all phosphorus fertilizer were added after 3- 4 pears of leave emerge on plant and the other half added after one month of the first. All plants received $60 \mathrm{~kg}$ ha- 1 potassium fertilizers as potassium sulfate. Weeds were controlled by hand and all agriculture practices were done as needed. Harvesting was done on $15^{\text {th }}$ June 2005 manually by pulling the dry plants out of the soil. Some physical and chemical properties of soil are measured in laboratory of horticulture department (table 1). The experiment was laid out in randomized complete block design with three replications. All measured Characters (plant height, stem diameter, number of branch per plant, fresh 
weight, number of fruit per plant, seed yield per plant, total seeds yield $\mathrm{kg} / \mathrm{ha}$ ) were subjected to the analysis of variance. And all data obtained were analyzed and compared statistically at a significance level of 5\%, using SAS program (SAS, 1996).

\begin{tabular}{|c|c|c|c|c|c|c|}
\hline $\mathrm{N}$ & $\mathrm{P}$ & $\mathrm{K}$ & $\mathrm{EC}$ & \multirow{2}{*}{$\mathrm{pH}$} & Organic mater & $\mathrm{CaCO}_{3}$ \\
\cline { 1 - 4 }$\%$ & $\%$ & $\%$ & mmhos/cm & & $\%$ & $\%$ \\
\hline 0.024 & 0.007 & 0.082 & 1.8 & 7.65 & 1.06 & 25.04 \\
\hline \multicolumn{2}{|c|}{ Clay $\%$} & \multicolumn{3}{|c|}{ Sand \% } & \multicolumn{3}{c|}{ Silt } & Texture \\
\hline \multicolumn{2}{|c|}{59.64} & & 23.4 & Silty sand \\
\hline
\end{tabular}

Table (1): some physical and chemical properties of soil

\section{RESULTS AND DISCUSSION}

Plant high: Data presented in table (2) showed that very high level of fertilizer $\left(320 \mathrm{~N}, 300 \mathrm{P}_{2} \mathrm{O}_{5}\right) \mathrm{kg} \mathrm{ha}^{-1}$ lead to significantly increased in plant high $5.72 \%$ when compared with high level of fertilizer $\left(280 \mathrm{~N}, 260 \mathrm{P}_{2} \mathrm{O}_{5}\right) \mathrm{kg} \mathrm{ha}^{-1}$, The reason may be due to very high nitrogen application which in itself increases plant growth then promoting processes such as cell division, cell enlargement, metabolic processes (George, 2000 ) or may be due to very high Phosphorus application which is important in plant bioenergetics as a component of ATP during photosynthesis Since ATP can be used for the biosynthesis of many plant bimolecular, also its use to modify the activity of various enzymes by phosphorylation, so phosphorus is important for plant growth and flower then seed formation (Wikipedia, 2007). Pinching case significantly decrease in plant high $38.14 \mathrm{~cm}$ when comparison with without pinching plants $42.00 \mathrm{~cm}$, these results are in agreement with those found by Cavins (2003), and Abdul Allah (2006). This may be relate to negative correlation between plant high and branches number( $\mathrm{r}=-0.027)$ as shown in table (10). Decreased seed rate sowing to $0.6 \mathrm{~g} / 10 \mathrm{~m}^{2}$ and increased it to $1.2 \mathrm{~g} / 10 \mathrm{~m}^{2}$ give plant high $40.50,41.16 \mathrm{~cm}$ respectively which significantly differ when comparison with those sowing at seed rate 1.0 , and $0.8 \mathrm{~g} / 10 \mathrm{~m}^{2} 39.11,39.49 \mathrm{~cm}$ respectively. The reason may be relate to few competition between plants when seed rate sowing are very few $0.6 \mathrm{~g} / 10 \mathrm{~m}^{2}$ and for elongation of plants when number of plants are very high due to increase seed rate sowing $1.2 \mathrm{~g} / \mathrm{m}^{2}$ (Hussen, 1981). Dual and triple interaction between the levels of studied factors has a significant influence on this character.

Stem diameter: Pinching and seed rate haven't any significant effect on stem diameter as shown in table (3), these results are in agreement with those found by Cavins (2003). Very high level of fertilizer have significant effect on this character $4.15 \mathrm{~mm}$ when compared with high level $3.90 \mathrm{~mm}$. these results are in agreement with those found by Ahmed (1997) and may refer to nitrogen and its role in create amino acids, which are the building blocks of proteins and forming protoplasm, the site for cell division and thus for plant growth and development then increased stem diameter. (Al-Naimi, 1987; Uchida, 2000). The interaction between fertilizer and pinching have a significant influence and the highest value $4.26 \mathrm{~mm}$ obtained in plants without pinch and fertilized with 
very high level fertilizer, other dual and third interactions haven't any effect except non pinched plant at sowing rate $0.6 \mathrm{~g} / 10 \mathrm{~m}^{2}$ and fertilized with very high level of fertilizer which superior significantly on pinching plant sowing at $0.8 \mathrm{~g} / 10 \mathrm{~m}^{2}$ seed rate and fertilized with high level.

Branch number: Very high level of nitrogen and phosphorus haven't any significant effect on branch number, as shown in table (4). Pinching effect significantly on this character $6.83 \mathrm{branch} / \mathrm{plant}$ when compared with non pinching $6.01 \mathrm{branch} / \mathrm{plant}$. This may be relate to lateral shoot development which be stimulated and promoted by either hand or chemical pinching as a result to remove inhibiter substance witch create in apical buds and transferred to lateral bud then inhibit ts sets and grown (Asiah et al. 1992; Phetpradap et al.1994).

Table(2):Effect of very high level of nitrogen and phosphorus fertilization, pinching, and Seed rate sowing on plant high $(\mathrm{cm})$ of Nigella sativa 1. plant

\begin{tabular}{|c|c|c|c|c|c|c|c|}
\hline \multirow{2}{*}{$\begin{array}{l}\text { Fertilizer } \\
\text { Levels }\end{array}$} & \multirow{2}{*}{ Pinch } & \multicolumn{4}{|c|}{ Seed sowing rate $\mathrm{g} / 10 \mathrm{~m}^{2}$} & Fertilizer & Fertilizer \\
\hline & & 1.2 & 1.0 & 0.8 & 0.6 & $\times$ pinch & effect \\
\hline \multirow{2}{*}{$\begin{array}{l}\text { Very } \\
\text { High }\end{array}$} & Pinch & $40.00 \mathrm{~b}-\mathrm{e}$ & $40.27 \mathrm{~b}-\mathrm{e}$ & $38.00 \mathrm{~d}-\mathrm{f}$ & $40.72 b-d$ & $39.75 b$ & \multirow{2}{*}{$41.18 \mathrm{a}$} \\
\hline & Without & $44.53 \mathrm{a}$ & $43.13 \mathrm{ab}$ & $41.75 \mathrm{a}-\mathrm{c}$ & $41.03 \mathrm{~b}-\mathrm{d}$ & $42.61 \mathrm{a}$ & \\
\hline \multirow{2}{*}{ High } & Pinch & $38.83 \mathrm{c}-\mathrm{f}$ & $33.92 \mathrm{~g}$ & $36.19 \mathrm{fg}$ & $37.15 \mathrm{ef}$ & $36.52 \mathrm{c}$ & \multirow{2}{*}{$38.95 b$} \\
\hline & Without & $41.29 \mathrm{a}-\mathrm{d}$ & $39.13 \mathrm{c}-\mathrm{f}$ & $42.00 \mathrm{a}-\mathrm{c}$ & $43.11 \mathrm{ab}$ & $41.38 \mathrm{a}$ & \\
\hline \multirow{2}{*}{$\begin{array}{c}\text { Fertilizer } \\
\times \\
\text { Seed } \\
\text { rate } \\
\text { sowing }\end{array}$} & $\begin{array}{l}\text { Very } \\
\text { High } \\
\end{array}$ & $42.27 \mathrm{a}$ & $41.70 \mathrm{ab}$ & $39.88 \mathrm{bc}$ & $40.88 \mathrm{a}-\mathrm{c}$ & \multirow{2}{*}{\multicolumn{2}{|c|}{$\begin{array}{l}\text { Pinch } \\
\text { effect }\end{array}$}} \\
\hline & High & $40.06 a-c$ & $36.53 c$ & $39.10 b c$ & 40.13a-c & & \\
\hline \multirow{2}{*}{$\begin{array}{l}\text { Pinch } \\
\times \\
\text { Seed } \\
\text { rate } \\
\text { sowing }\end{array}$} & Pinch & $39.42 \mathrm{bc}$ & $37.10 \mathrm{~d}$ & $37.10 \mathrm{~d}$ & $38.94 \mathrm{~cd}$ & \multicolumn{2}{|c|}{$38.14 \mathrm{~b}$} \\
\hline & Without & $42.91 \mathrm{a}$ & 41.13ab & $41.88 \mathrm{a}$ & $42.07 \mathrm{a}$ & \multicolumn{2}{|c|}{$42.00 \mathrm{a}$} \\
\hline Seed rate & wing & $41.16 \mathrm{a}$ & $39.11 b$ & $39.49 \mathrm{~b}$ & 40.50ab & & \\
\hline
\end{tabular}

Each means in row for one or interactions factors with different letters are significantly different at $\quad \mathrm{P}=0.05$ using Duncan's multiple range test.

Table(3):Effect of very high levels of nitrogen and phosphorus fertilization, pinching, and Seed rate sowing on stem diameter $(\mathrm{mm})$ of Nigella satival. plant.

\begin{tabular}{|c|c|c|c|c|c|c|c|}
\hline \multirow{2}{*}{$\begin{array}{c}\text { Fertilizer } \\
\text { Levels }\end{array}$} & \multirow{2}{*}{ Pinch } & \multicolumn{4}{|c|}{ Seed sowing rate $\mathrm{g} / 10 \mathrm{~m}^{2}$} & \multirow{2}{*}{$\begin{array}{l}\text { Fertilizer } \\
\times \text { pinch }\end{array}$} & \multirow{2}{*}{$\begin{array}{c}\text { Fertilizer } \\
\text { Effect }\end{array}$} \\
\hline & & 1.2 & 1.0 & 0.8 & 0.6 & & \\
\hline \multirow{2}{*}{$\begin{array}{l}\text { Very } \\
\text { High } \\
\end{array}$} & Pinch & $3.72 \mathrm{ab}$ & $3.93 \mathrm{ab}$ & $4.30 \mathrm{ab}$ & $4.25 \mathrm{ab}$ & $4.05 \mathrm{ab}$ & \multirow[b]{2}{*}{$4.15 \mathrm{a}$} \\
\hline & Without & $4.18 \mathrm{ab}$ & $4.22 \mathrm{ab}$ & $4.22 \mathrm{ab}$ & $4.42 \mathrm{a}$ & $4.26 \mathrm{a}$ & \\
\hline \multirow[b]{2}{*}{ High } & Pinch & $3.82 \mathrm{ab}$ & $4.14 \mathrm{ab}$ & $3.49 \mathrm{~b}$ & $3.74 \mathrm{ab}$ & $3.80 \mathrm{~b}$ & \multirow{2}{*}{$3.90 \mathrm{~b}$} \\
\hline & Without & $4.00 \mathrm{ab}$ & $3.83 \mathrm{ab}$ & $4.24 \mathrm{ab}$ & $3.92 \mathrm{ab}$ & $4.00 \mathrm{ab}$ & \\
\hline \multirow{2}{*}{$\begin{array}{c}\text { Fertilizer } \\
\times \\
\text { Seed rate } \\
\text { sowing } \\
\end{array}$} & $\begin{array}{l}\text { Very } \\
\text { High } \\
\end{array}$ & $3.95 \mathrm{a}$ & $4.08 \mathrm{a}$ & $4.26 \mathrm{a}$ & $4.34 \mathrm{a}$ & \multirow{2}{*}{\multicolumn{2}{|c|}{$\begin{array}{l}\text { Pinch } \\
\text { Effect }\end{array}$}} \\
\hline & High & $4.00 \mathrm{a}$ & $4.18 \mathrm{a}$ & $3.86 \mathrm{a}$ & $4.08 \mathrm{a}$ & & \\
\hline
\end{tabular}




\begin{tabular}{|c|c|c|c|c|c|c|}
\hline \multirow{2}{*}{$\begin{array}{c}\text { Pinch } \\
\times \\
\text { Seed rate } \\
\text { sowing }\end{array}$} & Pinch & $3.77 \mathrm{a}$ & $4.04 \mathrm{a}$ & $3.90 \mathrm{a}$ & $3.99 \mathrm{a}$ & $3.92 \mathrm{a}$ \\
\hline & Without & $4.09 \mathrm{a}$ & $4.03 \mathrm{a}$ & $4.23 \mathrm{a}$ & $4.17 \mathrm{a}$ & $4.13 \mathrm{a}$ \\
\hline \multicolumn{2}{|c|}{ Seed rate sowing } & $3.93 \mathrm{a}$ & $4.03 \mathrm{a}$ & $4.06 \mathrm{a}$ & $4.08 \mathrm{a}$ & \\
\hline
\end{tabular}

Each means in row for one or interactions factors with different letters are significantly different at $\mathrm{P}=0.05$ using Duncan's multiple range test.

Also decreased seed rate sowing cased significant increased in branch number ranged between $6.08-6.56$ branch/plant for seed rate sowing 1.2, 1.0, $0.8,0.6 \mathrm{~g} / 10 \mathrm{~m}^{2}$. All dual interaction between factors have significantly effect and the highest value 7.10 branch/plant obtained in pinched plants fertilized with very high level, so triple interaction have superior significantly on this characters and the highest value 7.52 branch/plant for pinching plant sowing at 0.8 seed rate and fertilized with high level .

Table(4):Effect of very high levels of nitrogen and phosphorus fertilization, pinching, and Seed rate sowing on branch number of Nigella sativa 1.

\begin{tabular}{|c|c|c|c|c|c|c|c|}
\hline \multirow{2}{*}{$\begin{array}{c}\text { Fertilizer } \\
\text { Levels }\end{array}$} & \multirow{2}{*}{ Pinch } & \multicolumn{4}{|c|}{ Seed sowing rate $\mathrm{g} / 10 \mathrm{~m}^{2}$} & \multirow{2}{*}{$\begin{array}{c}\text { Fertilizer } \\
\times \text { pinch }\end{array}$} & \multirow{2}{*}{$\begin{array}{c}\text { Fertilizer } \\
\text { Effect }\end{array}$} \\
\hline & & 1.2 & 1.0 & 0.8 & 0.6 & & \\
\hline \multirow{2}{*}{$\begin{array}{l}\text { Very } \\
\text { High }\end{array}$} & Pinch & $6.62 \mathrm{a}-\mathrm{c}$ & $7.13 \mathrm{ab}$ & $7.52 \mathrm{a}$ & $7.13 \mathrm{ab}$ & $7.10 \mathrm{a}$ & \multirow{2}{*}{$6.54 \mathrm{a}$} \\
\hline & Without & $5.54 \mathrm{e}$ & $5.80 \mathrm{c}-\mathrm{e}$ & $6.23 \mathrm{~b}-\mathrm{e}$ & $6.37 \mathrm{~b}-\mathrm{e}$ & $5.99 \mathrm{c}$ & \\
\hline \multirow{2}{*}{ High } & Pinch & $6.33 \mathrm{~b}-\mathrm{e}$ & $6.43 \mathrm{~b}-\mathrm{e}$ & $6.33 \mathrm{~b}-\mathrm{e}$ & $7.15 \mathrm{ab}$ & $6.56 \mathrm{~b}$ & \multirow{2}{*}{$6.30 \mathrm{a}$} \\
\hline & Without & $5.80 \mathrm{c}-\mathrm{e}$ & $6.57 \mathrm{a}-\mathrm{d}$ & $6.20 \mathrm{~b}-\mathrm{e}$ & $5.60 \mathrm{de}$ & $6.04 \mathrm{c}$ & \\
\hline \multirow{2}{*}{$\begin{array}{c}\text { Fertilizer } \\
\times \\
\text { Seed } \\
\text { rate } \\
\text { sowing } \\
\end{array}$} & $\begin{array}{l}\text { Very } \\
\text { High }\end{array}$ & $6.08 \mathrm{~b}$ & $6.46 \mathrm{ab}$ & $6.88 \mathrm{a}$ & $6.75 \mathrm{ab}$ & \multirow{2}{*}{\multicolumn{2}{|c|}{$\begin{array}{l}\text { Pinch } \\
\text { effect }\end{array}$}} \\
\hline & High & $6.07 \mathrm{~b}$ & $6.07 \mathrm{ab}$ & $6.07 \mathrm{ab}$ & $6.07 \mathrm{ab}$ & & \\
\hline \multirow{2}{*}{$\begin{array}{c}\text { Pinch } \\
\times \\
\text { Seed } \\
\text { rate } \\
\text { sowing }\end{array}$} & Pinch & $6.48 \mathrm{~b}-\mathrm{d}$ & $6.78 \mathrm{a}-\mathrm{c}$ & $6.93 \mathrm{ab}$ & $7.14 \mathrm{a}$ & \multicolumn{2}{|c|}{$6.83 \mathrm{a}$} \\
\hline & Without & $5.67 \mathrm{e}$ & $6.18 \mathrm{c}-\mathrm{e}$ & $6.22 \mathrm{c}-\mathrm{e}$ & $5.98 \mathrm{de}$ & \multicolumn{2}{|c|}{$6.01 \mathrm{~b}$} \\
\hline \multicolumn{2}{|c|}{ Seed rate sowing } & $6.08 \mathrm{~b}$ & $6.48 \mathrm{ab}$ & $6.57 \mathrm{a}$ & $6.56 \mathrm{a}$ & & \\
\hline
\end{tabular}

Each means in row for one or interactions factors with different letters are significantly different at $\mathrm{P}=0.05$ using Duncan's multiple range test.

Fresh weight: Data in table (5) showed that very high level of fertilizer lead to significantly increased in fresh weight $6.71 \mathrm{~g} / \mathrm{plant}$ when compared with high level $6.12 \mathrm{~g} / \mathrm{plant}$ with increments percentage $9.64 \%$. The reason may be refer to adequate nitrogen which promotes aerial vegetative growth, increases the top/root ratio, and essential for fruit and seed formation so affects both yield and quality characteristics of vegetable production (Okeno, 2001). Or to nitrogen and its effect on improves the quality and quantity of dry matter in leafy vegetables and protein (Tucker, 1999). Pinching also significantly increased fresh weight $6.69 \mathrm{~g} /$ plant when comparison with non pinched 6.14 $\mathrm{g} /$ plant. Decreased seed rate to $0.8 \mathrm{~g} / 10 \mathrm{~m}^{2}$ lead to significantly increase in 
fresh weigh from $5.96 \mathrm{~g} /$ plant for plants sowing at $1.2 \mathrm{~g} / 10 \mathrm{~m}^{2}$ to $6.72 \mathrm{~g} / \mathrm{plant}$ for plants sowing at $0.8 \mathrm{~g} / 10 \mathrm{~m}^{2}$. triple interaction have a significant influence

\begin{tabular}{|c|c|c|c|c|c|c|c|}
\hline \multirow{2}{*}{$\begin{array}{c}\text { Fertilizer } \\
\text { Levels }\end{array}$} & \multirow{2}{*}{ Pinch } & \multicolumn{4}{|c|}{ Seed sowing rate $\mathrm{g} / 10 \mathrm{~m}^{2}$} & \multirow{2}{*}{$\begin{array}{l}\text { Fertilizer } \\
\times \text { pinch }\end{array}$} & \multirow{2}{*}{$\begin{array}{c}\text { Fertilizer } \\
\text { effect }\end{array}$} \\
\hline & & 1.2 & 1.0 & 0.8 & 0.6 & & \\
\hline \multirow{2}{*}{$\begin{array}{l}\text { Very } \\
\text { High }\end{array}$} & Pinch & $7.26 \mathrm{a}$ & $7.44 \mathrm{a}$ & $7.11 \mathrm{a}$ & $6.85 \mathrm{ab}$ & $7.17 \mathrm{a}$ & \multirow{2}{*}{$6.71 \mathrm{a}$} \\
\hline & Without & $5.95 \mathrm{ab}$ & $6.32 \mathrm{ab}$ & $6.64 \mathrm{ab}$ & $6.11 \mathrm{ab}$ & $6.26 \mathrm{~b}$ & \\
\hline \multirow{2}{*}{ High } & Pinch & $5.34 \mathrm{~b}$ & $6.19 \mathrm{ab}$ & $6.69 \mathrm{ab}$ & $6.65 \mathrm{ab}$ & $6.22 \mathrm{~b}$ & \multirow{2}{*}{$6.12 \mathrm{~b}$} \\
\hline & Without & $5.28 \mathrm{~b}$ & $6.15 \mathrm{ab}$ & $6.43 \mathrm{ab}$ & $6.21 \mathrm{ab}$ & $6.02 \mathrm{~b}$ & \\
\hline \multirow{2}{*}{$\begin{array}{c}\text { Fertilizer } \\
\times \\
\text { Seed rate } \\
\text { sowing } \\
\end{array}$} & $\begin{array}{l}\text { Very } \\
\text { High }\end{array}$ & $6.61 \mathrm{a}$ & $6.88 \mathrm{a}$ & $6.88 \mathrm{a}$ & $6.48 \mathrm{a}$ & \multirow{2}{*}{\multicolumn{2}{|c|}{$\begin{array}{l}\text { Pinch } \\
\text { effect }\end{array}$}} \\
\hline & High & $5.31 \mathrm{~b}$ & $6.17 \mathrm{ab}$ & $6.56 \mathrm{a}$ & $6.43 \mathrm{a}$ & & \\
\hline \multirow{2}{*}{$\begin{array}{c}\text { Pinch } \\
\times \\
\text { Seed rate } \\
\text { sowing } \\
\end{array}$} & Pinch & $6.30 \mathrm{ab}$ & $6.81 \mathrm{a}$ & $6.90 \mathrm{a}$ & $6.75 \mathrm{a}$ & \multicolumn{2}{|c|}{$6.69 \mathrm{a}$} \\
\hline & Without & $5.61 \mathrm{~b}$ & $6.24 \mathrm{ab}$ & $6.54 \mathrm{ab}$ & $6.16 \mathrm{ab}$ & \multicolumn{2}{|c|}{$6.14 \mathrm{~b}$} \\
\hline \multicolumn{2}{|c|}{ Seed rate sowing } & $5.96 \mathrm{~b}$ & $6.52 \mathrm{ab}$ & $6.72 \mathrm{a}$ & $6.46 \mathrm{ab}$ & & \\
\hline
\end{tabular}

and the highest value $7.26 \mathrm{~g} /$ plant obtained in pinching plants fertilized with very high level of fertilizer and sowing at seed rate $1.2 \mathrm{~g} / 10 \mathrm{~m}^{2}$. All this results may be refer to significant and high significant effect for stem diameter, branch number, fruit number, and plant yield on this character as shown in correlation coefficient $(\mathrm{r}=0.478,0.301,0.499,0.447$ respectively) (table 9).

Table (5): Effect of very high levels of nitrogen and phosphorus fertilization, pinching, and Seed rate sowing on fresh weight (g)of Nigella sativa 1.

Each means in row for one or interactions factors with different letters are significantly different at $\mathrm{P}=0.05$ using Duncan's multiple range test.

Fruits number: Although very high level of fertilizer and seed rate haven't any significant effect on fruit number, as shown in table (6) Pinching has significant effect 29.94 fruit/plant when compared with non pinching 26.70 fruit/plant. The interaction between fertilizer levels and pinching have a significant influence and the highest value 30.55 fruit/plant obtained in plants pinched and fertilized with very high level of fertilizer were as the lowest value 25.81 fruit/plant obtained in non pinched plants fertilized with high level fertilizer. Other dual and triple interactions have significantly effect and the lowest value 21.87 fruit/plant obtained in non pinching plant which sowing at 1.2 seed rate and fertilized with high level. All superior results may be refer to high significant effect for stem diameter, branch number, and fresh weight on this character as in correlation coefficient $(\mathrm{r}=0.566,0.479,0.499$ respectively) (table 9).

Plant yield: Although pinching haven't any significant effect on plant yield as shown in table (7) Very high level of nitrogen and phosphorus has significant effect on this character $5.60 \mathrm{~g} / \mathrm{plant}$ when compared with high level 5.13 $\mathrm{g} /$ plant and the percentage of increase reach $9.16 \%$. This result is in agreement with Singh et al. , (1999.) and it may be refer to nitrogen which plants require it in large amounts and its a role in almost all plant metabolic processes so it 
effect on promotes rapid growth, increases leaf size and quality, hastens crop maturity, and promotes fruit and seed development. Or to phosphor which aids in root development, flower initiation, and seed and fruit development (Tucker, 1999; Uchida, 2000). The medium seed rate lead to significant increased in this character $5.84,5.72 \mathrm{~g} /$ plant for plant sowing at seed rate 1.0 and $0.8 \mathrm{~g} / 10 \mathrm{~m}^{2}$ respectively while highest $1.2 \mathrm{~g} / 10 \mathrm{~m}^{2}$ and lowest $0.6 \mathrm{~g} / 10 \mathrm{~m}^{2}$ sowing seed rate give lowest value $5.02,4.87 \mathrm{~g} / \mathrm{plant}$ respectively. The dual interaction between fertilizer levels and pinching have a significant influence on this characters and the highest value $5.82 \mathrm{~g} /$ plant for pinched plants fertilized with very high level of nitrogen and phosphorus fertilizer while lowest value 5.02 for non pinched plants fertilized with high level of nitrogen and phosphorus fertilizer. Other dual and triple interactions also have significant effect on this character. All superior results may be refer to significant and high significant effect for plant high stem diameter, and fresh weight of plant on this character as shown in correlation coefficient( $\mathrm{r}=0.288,0.356,0.447$ respectively) table 9$)$.

Table(6):Effect of very high levels of nitrogen and phosphorus fertilization, pinching, and Seed rate sowing on fruit number of Nigella sativa 1.

\begin{tabular}{|c|c|c|c|c|c|c|c|}
\hline \multirow{2}{*}{$\begin{array}{l}\text { Fertilizer } \\
\text { Levels }\end{array}$} & \multirow{2}{*}{ Pinch } & \multicolumn{4}{|c|}{ Seed sowing rate $\mathrm{g} / 10 \mathrm{~m}^{2}$} & \multirow{2}{*}{$\begin{array}{l}\text { Fertilizer } \\
\times \text { pinch }\end{array}$} & \multirow{2}{*}{$\begin{array}{c}\text { Fertilizer } \\
\text { effect }\end{array}$} \\
\hline & & 1.2 & 1.0 & 0.8 & 0.6 & & \\
\hline \multirow{2}{*}{ Very High } & Pinch & $29.68 \mathrm{ab}$ & $32.38 \mathrm{a}$ & $30.56 a b$ & $29.58 \mathrm{ab}$ & $30.55 \mathrm{a}$ & \multirow{2}{*}{$29.07 \mathrm{a}$} \\
\hline & Without & $29.63 \mathrm{ab}$ & $28.13 a-c$ & $27.20 \mathrm{a}-\mathrm{c}$ & $25.41 \mathrm{bc}$ & $27.60 \mathrm{ab}$ & \\
\hline \multirow{2}{*}{ High } & Pinch & $28.26 a-c$ & $31.23 \mathrm{ab}$ & $30.06 \mathrm{ab}$ & $27.75 a-c$ & $29.33 \mathrm{a}$ & \multirow{2}{*}{$27.57 \mathrm{a}$} \\
\hline & Without & $21.87 \mathrm{c}$ & $24.67 \mathrm{bc}$ & $29.33 \mathrm{ab}$ & $27.37 a-c$ & $25.81 \mathrm{~b}$ & \\
\hline \multirow{2}{*}{$\begin{array}{c}\text { Fertilizer } \times \\
\text { Seed rate } \\
\text { sowing }\end{array}$} & Very High & $29.66 \mathrm{a}$ & $30.26 \mathrm{a}$ & $28.88 \mathrm{ab}$ & $27.50 \mathrm{ab}$ & \multirow{2}{*}{\multicolumn{2}{|c|}{$\begin{array}{l}\text { Pinch } \\
\text { effect }\end{array}$}} \\
\hline & High & $25.06 \mathrm{~b}$ & 27.95ab & $29.70 \mathrm{a}$ & $27.56 a b$ & & \\
\hline \multirow{2}{*}{$\begin{array}{c}\text { Pinch } \\
\times \\
\text { Seed rate } \\
\text { sowing }\end{array}$} & Pinch & 28.97ab & $31.81 \mathrm{a}$ & $30.31 \mathrm{ab}$ & $28.66 \mathrm{ab}$ & \multicolumn{2}{|c|}{$29.94 a$} \\
\hline & Without & $25.75 b$ & $26.40 \mathrm{~b}$ & $28.27 \mathrm{ab}$ & $26.39 \mathrm{~b}$ & \multicolumn{2}{|c|}{$26.70 \mathrm{~b}$} \\
\hline \multicolumn{2}{|c|}{ Seed rate sowing } & $27.36 \mathrm{a}$ & $29.10 \mathrm{a}$ & $29.28 \mathrm{a}$ & $27.53 \mathrm{a}$ & & \\
\hline
\end{tabular}

Each means in row for one or interactions factors with different letters are significantly different at $\mathrm{P}=0.05$ using Duncan's multiple range test.

Table(7):Effect of very high levels of nitrogen and phosphorus fertilization, pinching, and Seed rate sowing on plant yield (g/plant)of Nigella sativa 1.

\begin{tabular}{|c|c|c|c|c|c|c|c|}
\hline \multirow{2}{*}{$\begin{array}{c}\text { Fertilizer } \\
\text { Levels }\end{array}$} & \multirow{2}{*}{ Pinch } & \multicolumn{4}{|c|}{ Seed sowing rate $\mathrm{g} / 10 \mathrm{~m}^{2}$} & \multirow{2}{*}{$\begin{array}{c}\text { Fertilizer } \\
\times \text { pinch }\end{array}$} & \multirow{2}{*}{$\begin{array}{c}\text { Fertilizer } \\
\text { effect }\end{array}$} \\
\hline & & 1.2 & 1.0 & 0.8 & 0.6 & & \\
\hline \multirow{2}{*}{$\begin{array}{l}\text { Very } \\
\text { High }\end{array}$} & Pinch & $5.92 \mathrm{a}$ & $5.96 \mathrm{a}$ & $6.02 \mathrm{a}$ & $5.39 \mathrm{ab}$ & $5.82 \mathrm{a}$ & \multirow{2}{*}{$5.60 \mathrm{a}$} \\
\hline & Without & $5.15 \mathrm{ab}$ & $5.70 \mathrm{ab}$ & $5.92 \mathrm{a}$ & $4.73 \mathrm{ab}$ & $5.37 \mathrm{ab}$ & \\
\hline \multirow{2}{*}{ High } & Pinch & $4.53 \mathrm{~b}$ & $5.85 \mathrm{a}$ & $5.60 \mathrm{ab}$ & $4.97 \mathrm{ab}$ & $5.24 \mathrm{ab}$ & \multirow{2}{*}{$5.13 \mathrm{~b}$} \\
\hline & Without & $4.46 \mathrm{~b}$ & $5.86 \mathrm{a}$ & $5.36 \mathrm{ab}$ & $4.40 \mathrm{~b}$ & $5.02 \mathrm{~b}$ & \\
\hline \multirow{2}{*}{$\begin{array}{c}\text { Fertilizer } \\
\times \\
\text { Seed rate } \\
\text { sowing }\end{array}$} & $\begin{array}{l}\text { Very } \\
\text { High }\end{array}$ & $5.53 \mathrm{a}-\mathrm{c}$ & $5.83 \mathrm{ab}$ & $5.97 \mathrm{a}$ & $5.06 \mathrm{~b}-\mathrm{d}$ & \multirow{2}{*}{\multicolumn{2}{|c|}{$\begin{array}{l}\text { Pinch } \\
\text { effect }\end{array}$}} \\
\hline & High & $4.50 \mathrm{~d}$ & $5.86 \mathrm{ab}$ & $5.48 \mathrm{a}-\mathrm{c}$ & $4.69 \mathrm{~cd}$ & & \\
\hline
\end{tabular}




\begin{tabular}{|c|c|c|c|c|c|c|}
\hline \multirow{2}{*}{$\begin{array}{c}\text { Pinch } \\
\times \\
\text { Seed rate } \\
\text { sowing }\end{array}$} & Pinch & $5.23 \mathrm{a}-\mathrm{c}$ & $5.90 \mathrm{a}$ & $5.81 \mathrm{a}$ & $5.18 \mathrm{a}-\mathrm{c}$ & $5.53 \mathrm{a}$ \\
\hline & Without & $4.81 \mathrm{bc}$ & $5.78 \mathrm{a}$ & $5.64 \mathrm{ab}$ & $4.56 \mathrm{c}$ & $5.20 \mathrm{a}$ \\
\hline \multicolumn{2}{|c|}{ Seed rate sowing } & $5.02 \mathrm{~b}$ & $5.84 \mathrm{a}$ & $5.72 \mathrm{a}$ & $4.87 \mathrm{~b}$ & \\
\hline
\end{tabular}

Each means in row for one or interactions factors with different letters are significantly different at $\mathrm{P}=0.05$ using Duncan's multiple range test.

Total seeds yield $\mathbf{k g} / \mathbf{h a :}$ Data in table (8) show that total seed yield cannot effected significantly with pinching while very high level of nitrogen and phosphorus fertilizer have significant effect on this character $1010.4 \mathrm{~kg} / \mathrm{ha}$ when compared with high level $891.9 \mathrm{~kg} / \mathrm{ha}$ and the percentage of increase reach 13.29\%. This result is in agreement with Hammo and Al- Atractii, 2006 and Ahmed, 1997. Increased seed rate sowing from 0.6 to $1.2 \mathrm{~g} / 10 \mathrm{~m}^{2}$ lead to significant increased in this character from 651.9 to $1232.7 \mathrm{~kg} / \mathrm{ha}$ respectively and the highest increase percentage between high and low value reach $89.10 \%$. These results are in agreement with those found by Ghosh et al., (1981) which found that large distant between nigella plants may decrees total yield for unit area. The main reason for total seeds yield refer to plants number in unit area which increase with seed sowing rate increase. With increase seed sowing rate the interaction between fertilizer and pinching have a significant influence on this characters and the highest value $1078.7 \mathrm{~kg} / \mathrm{ha}$ for pinched plants fertilized with very high level of nitrogen and phosphorus fertilizer while lowest value $876.7 \mathrm{~kg} / \mathrm{ha}$ for non pinched plants fertilized with high level of nitrogen and phosphorus fertilizer. Other interactions also have significant effect on this characters, and the highest plant yield for triple interaction are $1454.8 \mathrm{~kg} / \mathrm{ha}$ for pinched plant sowing at seed rate $1.2 \mathrm{~g} / 10 \mathrm{~m}^{2}$ and fertilized with very high level fertilizer while the lowest value $540.7 \mathrm{~kg} / \mathrm{ha}$ obtained in non pinched plant sowing at seed rate $0.6 \mathrm{~g} / 10 \mathrm{~m}^{2}$ and fertilized with high level fertilizer. All superior results may be refer to high significant effect for branches number, plant yield on this character as shown in correlation coefficient $(\mathrm{r}=0.399,0.531$, respectively) table (9) .

Table(8):Effect of very high levels of nitrogen and phosphorus fertilization, pinching, and Seed rate sowing on total seed yield (kg/ hectare) of Nigella sativa 1.

\begin{tabular}{|c|c|c|c|c|c|c|c|}
\hline \multirow{2}{*}{$\begin{array}{c}\text { Fertilizer } \\
\text { Levels }\end{array}$} & \multirow{2}{*}{ Pinch } & \multicolumn{4}{|c|}{ Seed sowing rate $\mathrm{g} / 10 \mathrm{~m}^{2}$} & \multirow{2}{*}{$\begin{array}{c}\text { Fertilizer } \\
\times \text { pinch } \\
\end{array}$} & \multirow{2}{*}{$\begin{array}{c}\text { Fertilizer } \\
\text { Effect }\end{array}$} \\
\hline & & 1.2 & 1.0 & 0.8 & 0.6 & & \\
\hline \multirow{2}{*}{$\begin{array}{l}\text { Very } \\
\text { High } \\
\end{array}$} & Pinch & $1454.8 \mathrm{a}$ & $1098.0 \mathrm{c}-\mathrm{d}$ & $886.8 \mathrm{c}-\mathrm{e}$ & $875.3 \mathrm{c}-\mathrm{e}$ & $1078.7 \mathrm{a}$ & \multirow{2}{*}{$1010.4 \mathrm{a}$} \\
\hline & Without & $1265.8 \mathrm{ab}$ & $1049.7 \mathrm{~b}-\mathrm{d}$ & $872.4 \mathrm{c}-\mathrm{e}$ & $580.7 \mathrm{ef}$ & $942.1 \mathrm{ab}$ & \\
\hline \multirow{2}{*}{ High } & Pinch & $1114.1 \mathrm{bc}$ & $1078.3 \mathrm{~b}-\mathrm{d}$ & $825.2 \mathrm{c}-\mathrm{f}$ & $610.7 \mathrm{ef}$ & $907.1 \mathrm{~b}$ & \multirow{2}{*}{$891.9 \mathrm{~b}$} \\
\hline & Without & $1096.1 \mathrm{~b}-\mathrm{d}$ & $1080.1 \mathrm{~b}-\mathrm{d}$ & 789.9 d-f & $540.7 \mathrm{f}$ & $876.7 \mathrm{~b}$ & \\
\hline \multirow{2}{*}{$\begin{array}{c}\text { Fertilizer } \\
\times \\
\text { Seed rate }\end{array}$} & $\begin{array}{l}\text { Very } \\
\text { High }\end{array}$ & $1360.3 \mathrm{a}$ & $1073.8 \mathrm{bc}$ & $879.6 \mathrm{~cd}$ & $728.0 \mathrm{de}$ & \multirow{2}{*}{\multicolumn{2}{|c|}{$\begin{array}{l}\text { Pinch } \\
\text { Effect }\end{array}$}} \\
\hline & High & $1105.1 \mathrm{~b}$ & $1079.19 \mathrm{bc}$ & $807.5 \mathrm{~d}$ & $575.7 \mathrm{e}$ & & \\
\hline
\end{tabular}


Mesopotamia J. of Agric.

(ISSN 1815-316X)

Vol. (36) No. (1)

2008

\begin{tabular}{|c|c|c|c|c|c|c|}
\hline sowing & & & & & & \\
\hline $\begin{array}{c}\text { Pinch } \\
\times\end{array}$ & Pinch & $1284.4 \mathrm{a}$ & $1088.1 \mathrm{ab}$ & $856.01 \mathrm{c}$ & $743.0 \mathrm{~cd}$ & $992.9 \mathrm{a}$ \\
\hline $\begin{array}{c}\text { Seed rate } \\
\text { sowing }\end{array}$ & Without & $1180.9 \mathrm{ab}$ & $1064.9 \mathrm{~b}$ & $831.1 \mathrm{c}$ & $560.7 \mathrm{~d}$ & $909.4 \mathrm{a}$ \\
\hline \multicolumn{2}{|c|}{ Seed rate sowing } & $1232.67 \mathrm{a}$ & $1076.51 \mathrm{~b}$ & $843.56 \mathrm{c}$ & $651.85 \mathrm{~d}$ & \\
\hline
\end{tabular}

Each means in row for one or interactions factors with different letters are significantly different at $\mathrm{P}=0.05$ using Duncan's multiple range test. 
Table (9): correlation coefficients between characters

\begin{tabular}{|l|c|c|c|c|c|c|}
\hline characters & $\begin{array}{c}\text { Plant } \\
\text { high }\end{array}$ & $\begin{array}{c}\text { Stem } \\
\text { diameter }\end{array}$ & $\begin{array}{c}\text { Branches } \\
\text { number }\end{array}$ & $\begin{array}{c}\text { Fresh } \\
\text { weight }\end{array}$ & $\begin{array}{c}\text { fruits } \\
\text { number }\end{array}$ & $\begin{array}{c}\text { Total seeds } \\
\text { yield kg ha-1 }\end{array}$ \\
\hline Stem diameter & $0.29 *$ & & & & & \\
\hline Branch number & -0.027 & 0.244 & & & & \\
\hline Fresh weight & 0.249 & $0.478^{* *}$ & $0.301^{*}$ & & & \\
\hline fruits number & 0.226 & $0.566^{* *}$ & $0.479^{* *}$ & $0.499 * *$ & & \\
\hline Plant yield & $0.288^{*}$ & $0.356^{*}$ & 0.22 & $0.447^{* *}$ & 0.221 & \\
\hline $\begin{array}{l}\text { Total seeds } \\
\text { yield kg ha }\end{array}$ & 0.281 & 0.192 & $0.399^{* *}$ & 0.234 & 0.207 & $0.531^{* *}$ \\
\hline
\end{tabular}

تأثير المستويات العالية من السماد النتروجيني والفوسفاتي، القرط، ونسبة البذور المزروعة في النمو الخضري ومكونات البذور لنبات حبة البركة (Nigella sativa L)

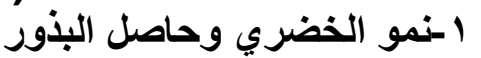

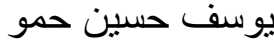

قسم البستنة / كلية الزر اعة / جامعة دهوك / العراق

\section{الخلاصة}

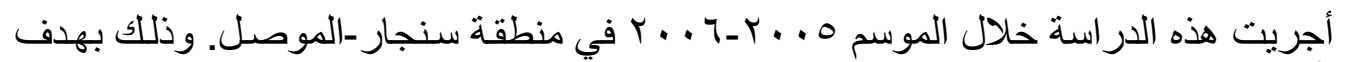

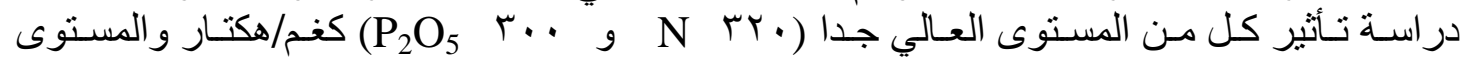

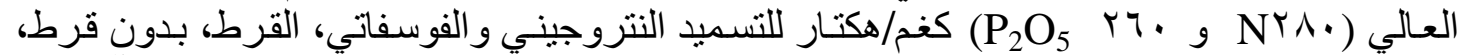

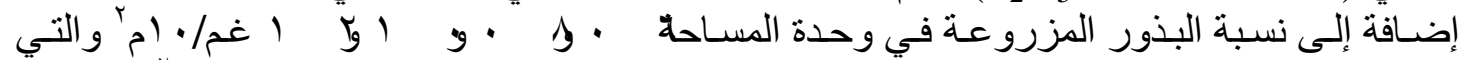

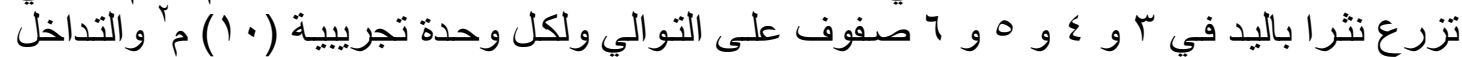
بينهما في نمو وحاصل البذور لنبات حبة البركة. نفذت التجربة باستخدام تصميم القطاعات العشو ائية

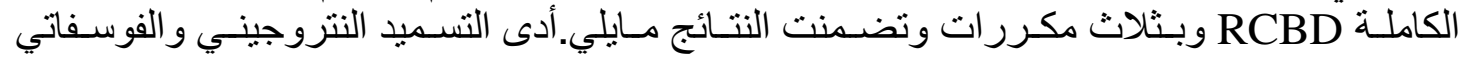

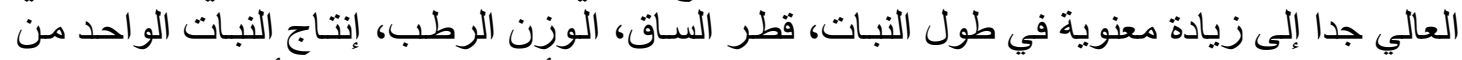

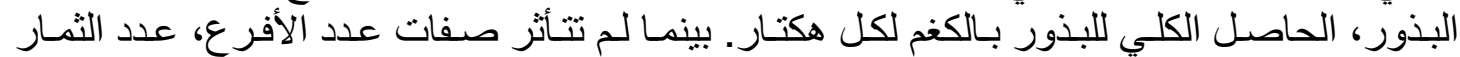

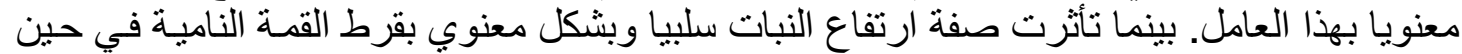

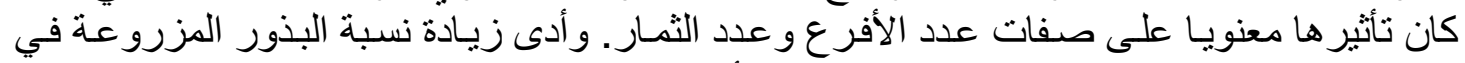

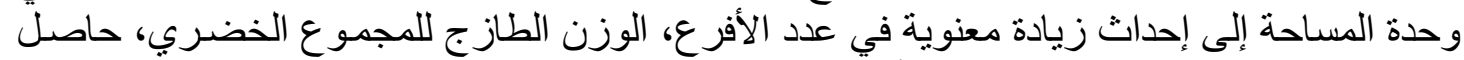

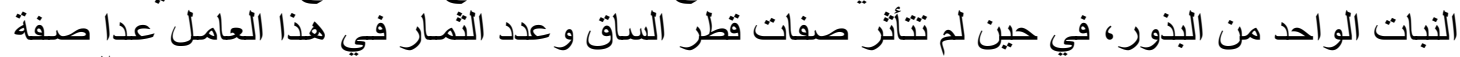

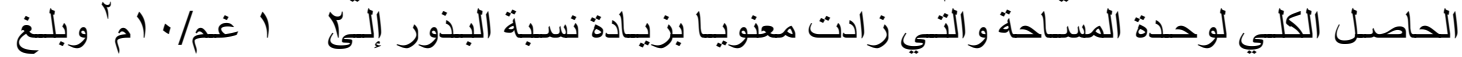

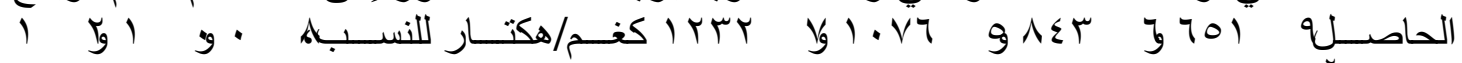

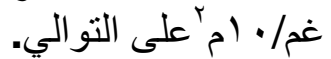

\section{REFERENCES}

Abdul Allah, B. Z. A (2006). Effect of Some Treatments on Growth and Flowering of (Cinerari Pericallis $X$ hybrida). Master thesis, College of Agriculture and Forestry, University of Mosul, Iraq.

Abu Zaid, A. N. (1986).Medicinal herbaceous and plants. Madboli library, Cairo.(Arabic)

Ahmed, E. T. (1997). Influence of plant distance and some phosphorous fertilization sources on black cumin (Nigella sativa L.) plants. Assiut .J. Agric. Sick. 28 (2):498-505. 
Al-Dagawi, A (1996). Aromatic and medicinal plant introduction encyclopedia. Madboli library. Cairo. Egypt.

Al-Khatony, Y. H. H. M. (2003). Effect of Some Agricultural Factors on Growth, Yield and Oil of Nigella sativa L. plants. ph.D. Thesis. College Of Agriculture and Forest University of Mosul, Iraq.

Al-Naimi, S. N. (1987) .Fertilizer and soil fertility. Ministry of high education and scientific research. Mosul University, Iraq.

Anderson, N. and W. Peters (2002). Potted plant production of (Gaura lindheimeri). Commercial Flower Growers Bulletin, April (2002) Page 7.

Atta, M. B (2003). Some characteristics of Nigella (Nigella sativa L.) seed cultivated in Egypt and its lipid profile. Food Chem., 83: 63-8.

Cavins, T. J., L. Greer, J. L; Gibson, B. E. Whipker, and J. M. Dole (2003). Response of marguerite daisy (Argyranthemum frutescens) "Comet Pink" to plant growth regulators. PGRSA Quarterly. 31(1): 2-7.

Ecke, P. and O. A. Matkin (1976). The Poinsettia manual. Encinitas, California, United States, Paul Ecke Poinsettias.

El-Kadi, A., and O. Kandil (1987). The black seed (Nigella sativa L.) and immunity: its effect on human cell subset. Fed. Proc., 46: 12-22.

George, O (2001). Effect of container volume and nitrogen application on the growth of young citrus seedlings Proceedings of the Horticulture seminar on Sustainable Horticultural Production in the tropics October 3rd to 6th 2001. Jomo Kenyatta University of Agriculture and Technology, JKUAT, Juja, Kenya.

Ghosh, D.; K. Roy and S. C. Malik (1981). Effect of fertilizers and spacing on yield and other characters of black cumin Nigella sativa. Indian Agriculturist 25 (3): 191 - 197 (C. F. Hort . Abst. 52 (8),:5666 ).

Hammo, Y. H. and A.. O. Al-Atrakchii (2006). Effect of nitrogen, phosphorus fertilizers and plant distances on growth of (Nigella sativa L.).1Vegetative growth and seed oil production. Mesopotamia J. of Agric. 34 (3) $2006 \cdot \mathrm{p}$ 17-26.

Hanafy, M. S. M. and M. E. Hatem (1991). Studies on the antimicrobial activity of (Nigella sativa L.) seed (black cumin). J. Ethnoph., 34: 275-8.

Larson, R. A (1980). Introduction to horticulture. United States, Academic Press, Inc.

Love, J. W. (1975). Vegetative growth In: Growing azaleas commercially. Kofranek, A. M.; Larson, R. A. ed. University of California, United States, Division of Agricultural Science.

Majed, S. H, and M. G. Mahmod (1988). Plants and Iraq herbaceous between folk medicine and scientifically research .Dar Althea for journalism and publication. Baghdad .Iraq.

Okeno, J. A. (2001). Effect of nitrogen for sustainable vegetable production: recent developments. Proceedings of the Horticulture seminar on Sustainable Horticultural Production in the tropics October 3rd to 6th 2001. Jomo Kenyatta University of Agriculture and Technology, Jkuat, Juja, Kenya. 
Phetpradap, S, J. G. Hamptoni and M. J. Hill (1994). Effect of hand pinching and plant growth regulators on seed production of field grown hybrid dahlia. New Zealand Journal of Crop and Horticultural Science, 1994, 22: 313-320.

SAS (1989-1996). Proprietary soft ware release, 6.12 TS 020 Licensed to North Carolina state university. By SAS Institute Inc., Cary. NC. USA.

Singh, S. K.; S. Sardar and S. Singh (1999). Response of Nigella (Nigella sativa) to nitrogen and phosphorous Crop. Res. Hisar. 1999: 18 (3). (C. F. CAB Abstracts 1998 / 08 - 2000/04).

Tierra, M. L. AC. OMD (2002). Online articles. (Nigella sativa L.) Commonly known as "love in the mist" www. Planetherbe, com /articles/nigella. htm1.

Tucker, M. R (1999). Essential plant nutrients: their presence in North Carolina soils and role in plant nutrition. Agronomic Division, NCDA \& CA October: 1-9.

Uchida, R. (2000). Plant nutrient management in Hawaii's Soils, Approaches for Tropical and Subtropical Agriculture J. A. Silva and R. Uchida, Eds. College of Tropical Agriculture and Human Resources, Hawaii University

Wikipedia, the free encyclopedia. (2007).Plant nutrition.GNU Free Documentation License 501(c)(3) tax-deductible nonprofit charity, 22:35,5 october. 